\title{
Bilateral Galcancal Fractures in a Twelve Year Old Boy
}

\section{Fatih Türkmensoy, Kaya Akan, Oguz Poyanli, Bahattin Kemah, Samet Erinc From the Department of Orthopaedics and Traumatology, Istanbul Civilisation University Goztepe Research and Training Hospital, Turkey.}

\section{Abstract:}

Calcaneal fractures generally occur with high energy trauma. These fractures are more common in adults and men are affected more than women. In childhood incidence of these fractures is very rare. In this case report, we aimed to present a bilateral calcaneal fracture in a twelve year old boy and its potential injury mechanism.

Key words: Calcaneus, Fractures, Foot Injuries, Ankle Injuries, Humans.

\section{Introduction}

Calcaneal fractures are rare in childhood [1]. Although the scientific literature is supporting operative management of calcaneal fractures in adults, there are few studies in children as calcaneal fractures are rare in this age group $[2,3]$. While calcaneal fractures constitute 1 to $2 \%$ of all fractures in adulthood, this occurrence rate drops sharply to $0.005 \%$ in childhood [3]. In this case, we report a 12 year-old-boy with bilateral calcaneal fracture, due to jump from height.

\section{Case Report}

A twelve year-old-boy was admitted to the Emergency Unit with bilateral heel and ankle pain. He jumped wearing a track shoe from a wall, which is approximately three to four meters, to ground while he was running away from a dog attack. His initial examination revealed an edema and ecchymosis starting from hindfoot extending through his plantar arch on his both feet. Bilateral tenderness was detected with palpation on his ankles and heels. Integrity of skin was intact on his both feet. On his systemic examination no other abnormality was detected. The initial trauma X-ray series which included bilateral feet, ankles, knees, pelvis and vertebral column, detected only bilateral calcaneal fractures without any bony involvement. Further workups including CT scans for both feet and 3D reconstructions were performed to understand the fracture characteristics. A long leg casts were applied in both lower extremities for temporary procedure with elevation in the bed.

The measurements of both calcaneus were made. The Böhler angle was $27.42^{\circ}(20-45)$ [Fig. 1] on the right side and the $34.81^{\circ}$ [Fig.3] on the left side. The Gissane crucial angle was $111.90^{\circ}$ (120-145)

Corresponding Author: Dr. Fatih Türkmensoy

Email: turkmensoyfatih@yahoo.com

Received: August 20, 2013 | Accepted: October 11, 2013 | Published Online: October 25, 2013

This is an Open Access article distributed under the terms of the Creative Commons Attribution License (creativecommons.org/licenses/by/3.0)

Conflict of interest: None declared | Source of funding: Nil | DOl: http://dx.doi.org/10.17659/01.2013.0087 
[Fig.2] on the right side and $129.97^{\circ}$ [Fig.4] on the left side. Fractures were extending to subtalar joints on both sides. The angles of left side were considered within conservative therapy limits. Therefore, a long leg cast was accepted for the definitive treatment forthis side. A closed manipulation with the wellknown Essex-Lopresti procedure was planned on the right foot. The patient was operated on the third day after taking informed consent.

After closed reduction under general anesthesia the Essex-Lopresti procedure was performed by a $3.5 \mathrm{~mm}$ Schanz pin which held the calcaneus in reduced position. The accepted position was confirmed with fluoroscopy. A pin incorporated long leg cast was applied to the lower extremity.

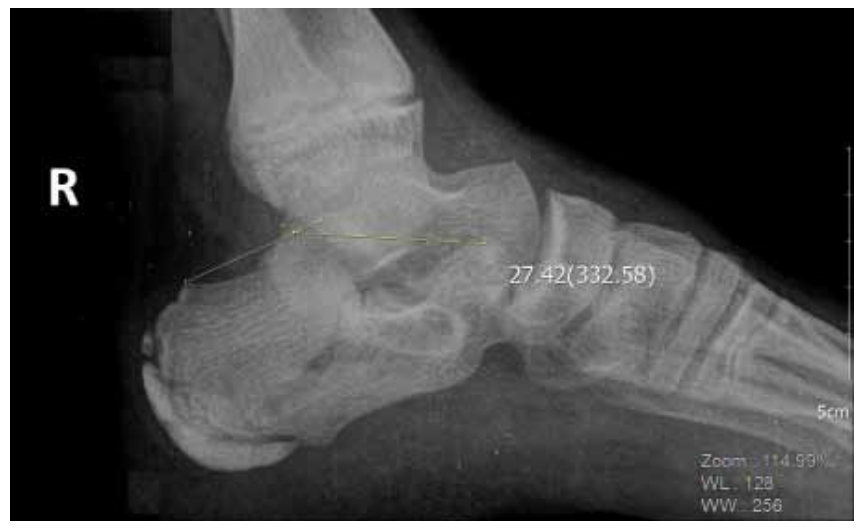

Fig.1: Preoperative right side Böhler's angle: $27.42^{\circ}$.

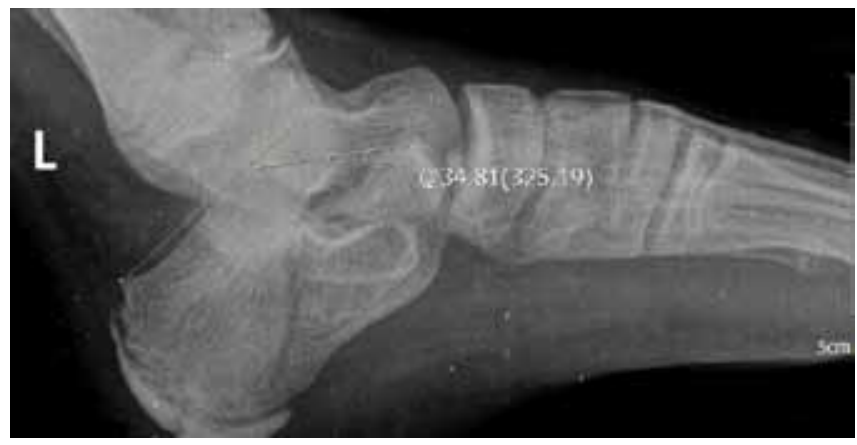

Fig.3: Preoperative left side Böhler's angle: $34.81^{\circ}$.
The postoperative $\mathrm{X}$-rays and $\mathrm{CT}$ scans revealed that the Böhler angle was $39.04^{\circ}$ [Fig.5] and the Gissane crucial angle was $125.09^{\circ}$ [Fig.6] on the right side. His both extremities were elevated in bed. He was discharged on seventh day. A control $\mathrm{X}$-ray on postoperative third week showed no loss of reduction. The casts were removed at sixth week with the Schanz pin under local anesthesia. Physical therapy was started immediately after cast removal and the patient was encouraged to weight-bear with two crutches for another two weeks. Until the second month, full weight bearing was not allowed with follow up planned every two months. After four months he could walk unlimited withoutany pain. His $X$-rays after one year showed good healing with the Böhler and the Gissane crucial angles $35^{\circ}$ and $123^{\circ}$ respectively on operated side and his AOFAS score was 100 for both feet.

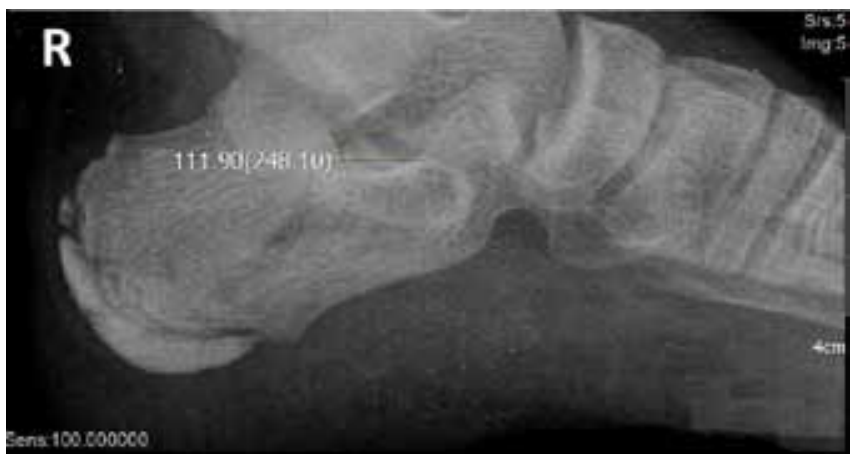

Fig.2: Preoperative right side Gissane angle: $111.90^{\circ}$.

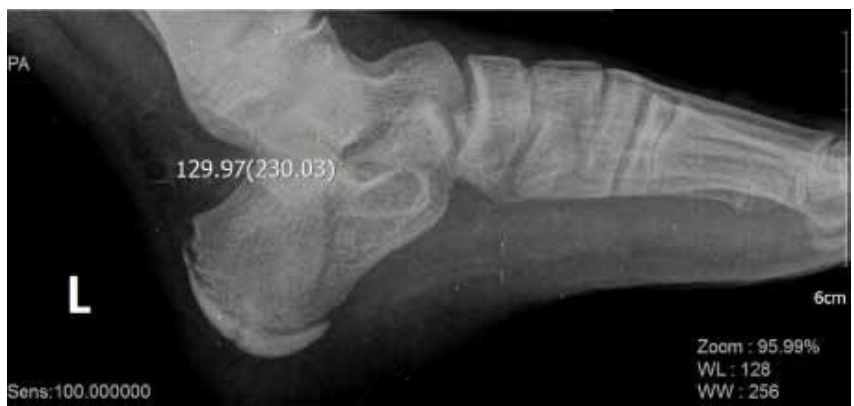

Fig.4: Preoperative left side Gissane angle: $129.97^{\circ}$. 


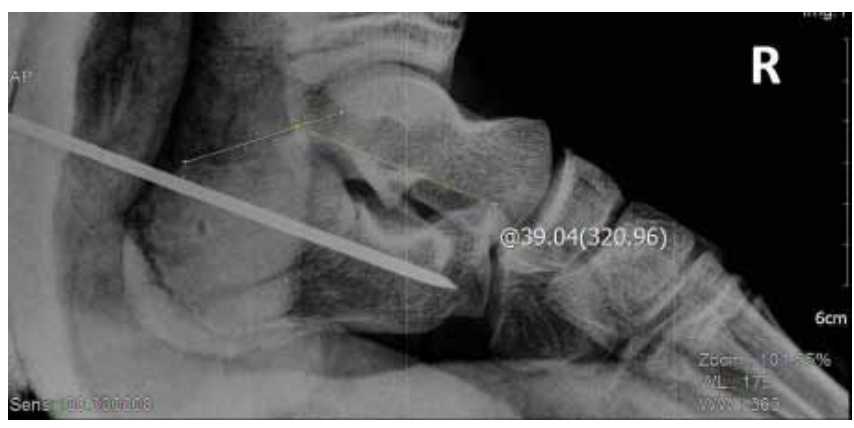

Fig.5: Postoperative right side Böhler's angle: $39.04^{\circ}$.

\section{Discussion}

Calcaneal fractures are rare in children less than 18 years of age with incidence estimated to be between $0.005-0.41 \%$. Displaced calcaneal fractures are even more rare in pediatric population [4-6]. Calcaneal fractures are most often the result of low-energy injuries in children, and the fractures are often undisplaced or minimally displaced [6]. Children's feet have a relative strength of the bones in their feet in relation to body weight and they have a larger cartilage component preventing displacement of fractures. The increased elasticity of the pediatric skeleton and cartilage results in less stress on the calcaneus [6]. The rate of delayed diagnosis of pediatric calcaneal fractures is estimated at $27-55 \%$ [5]. History of fall or jump with heel pain should arouse suspicion of calcaneal fracture and CT scan should be performed in case of suspicion [3]. In addition to the trauma series, the $X$-rays of the tender areas should be taken since these patients are prone to have other injuries.

Treatments of calcaneal fractures conservatively in childhood are discussed by many authors in the literature, also for both of intra- and extra-articular fractures [5]. In pediatric calcaneal fractures nonoperative treatment provide good results for following reasons: strong subchondral bone resists significant fracture displacement in children, the

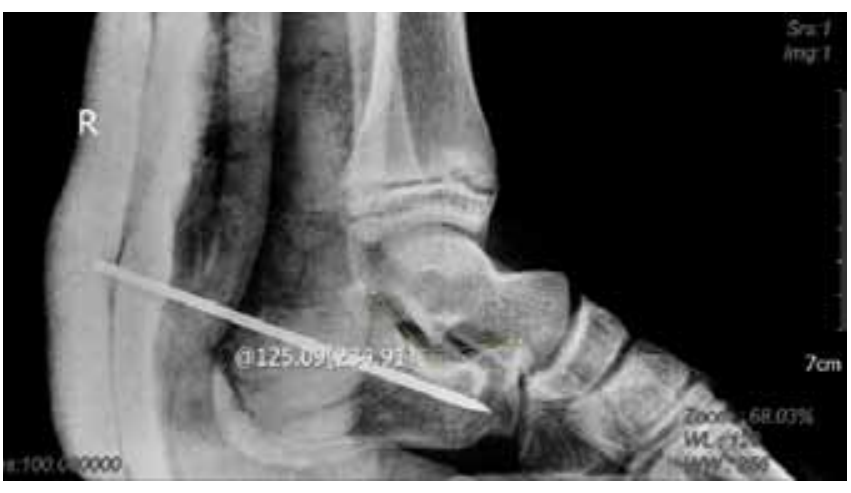

Fig.6: Postoperative right side Gissane angle: $125.09^{\circ}$.

formation of fractures with low-energy trauma, the lateral process of the talus is small and the posterior subtalar facet is parallel to the ground in children, and the injuring force through the lateral process of the talus is distributed throughout a wider area than in adults [6].

Open treatment of calcaneal fractures in children was advocated in literature when the fracture is displaced or fracture is open fracture. Surgery options include wide range like percutaneous (semiopen) technique according to the Essex-Lopresti method or by open reduction using the extensile lateral exposure and fixation with a plate [7].

Herein, we reported a rare case of 12 year-old boy with isolated bilateral calcaneal fracture after jump from height. Soft tissue complications for open reduction and internal fixation are similar to adults in calcaneal fractures presenting with high energy trauma and as well as lumber vertebral column, knee and talus fractures $[8,9]$. Our case had only isolated bilateral calcaneal fractures although with high energy trauma. We thought it was related with controlled jump. Probably first his forefoot came in contact with ground first and absorbed maximum energy which was followed by contact of heel with ground causing an undisplaced calcaneal fracture. 


\section{Conclusion}

Fractures of calcaneus in children can occur with low or high-energy trauma. Choice of the treatment for pediatric calcaneal fractures depends on the patient's age, fracture type, displacement, and soft tissue situation. The major factors which determine the fracture characteristics are amount of total energy and direction of trauma to calcaneus.

\section{References}

1. Schantz K, Rasmussen F. Calcaneus fracture in the child. Acta Orthop Scand. 1987;58:507509.

2. Summers $\mathrm{H}$, Kramer PA, Benirschke SK. Pediatric calcaneal fractures. Orthopedic Reviews. 2009; 1:30-33.

3. Frank EV, Ward JC, Engelhard P. Bilateral calcaneal fracture in childhood. Arch Orthop Trauma Surg.1998;118:111-112.
4. de Beer JD, Maloon S, Hudson DA. Calcaneal fractures in children. S Afr Med J. 1989;76:5354.

5. Dudda M, Kruppa C, Gebmann J, Seybold D, Schildhaver TA. Pediatric and adolescent intra articular fractures of the calcaneus. Orthop Rev (Pavia). 2013;5:82-85.

6. Yu GR, Zhao HM, Yang YF, Zhou JQ, Li HF. Open reduction and internal fixation of intra-articular calcaneal fractures in children. Orthopedics. $2012 ; 35: 874-879$.

7. Brunet JA. Calcaneal fractures in children. J Bone Joint Surg Br. 2000;82:21 1-216.

8. Beaty JH, Kasser JR. Fractures and dislocations of the foot. In:Beaty JH, Kasser JR(eds). Rockwood \& Wilkins' Fractures in Children, $6^{\text {th }}$ Edition. Philadelphia: Lippincott Williams and Wilkins2006. pp.1147-1148.

9. Bahari S, McKenna J. Subtalar and Extra Articular Synovial Chondromatasis. Journal of Case Reports. 2012;2:1 20-124. 